\title{
ON SOME PROPERTIES OF MONIC POLYNOMIALS
}

\author{
P. BHATTACHARYYA
}

\section{Introduction}

Let

$$
f(z)=z^{k}+a_{1} z^{k-1}+\cdots+a_{k}, \quad k \geq 2
$$

be a monic polynomial of degree $k$.

Let $E\{z:|f(z)| \leq R\}$ where $R>0$ and $d(E)$ be the diameter of the set $E$. Then we prove

Theorem 1.

$$
d(E) \geq 2 R^{1 / k}
$$

The theory of iteration of a rational or entire function of a complex variable $z$ deals with the sequence of natural interates $f_{n}(z)$ defined by

$$
f_{0}(z)=z, f_{n+1}(z)=f\left(f_{n}(z)\right), n=0,1, \cdots \text {. }
$$

In the theory developed by Fatou [3,4] and Julia [5], the central object of study is the Fatou Set of those points of the complex plane in no neighbourhood of which the sequence of natural interates $\left\{f_{n}(z)\right\}$ forms a normal family in the sense of Montel. Unless $f(z)$ is a rational function of order 0 or 1 , the set $F(f)$ is a nonempty perfect set. For a survey of the main properties of the Fatou Set we refer the reader to the excellent paper of Brolin [2]. Bhattacharyya and Arumaraj [1] considered the question of bounds of the diameter of the set $F(f)$ where $f(z)$ is a monic polynomial. They conjectured that if $f(z)$ is a monic polynomial of degree $\geq 2$, then $d(F(f)) \geq 2$.

We will show that this conjecture follows from our Theorem 1 . We thus have

Theorem 2. If $f(z)$ is a monic polynomial of degree $k \geq 2$, then $d(F(f)) \geq 2$.

We also prove

Theorem 3. If $d(F(f))=2$ where $f$ is a monic polynomial of degree $k \geq 2$, then $f(z)=(z-\alpha)^{k}, k \geq 2$ for some $\alpha$ which can be zero.

Received June 20, 1989. 
If $\alpha$ is zero then it is obvious that $d\left(F\left(z^{k}\right)\right)=2$. This shows that the bounds obtained in theorem 1 and 3 are sharp.

We prove our theorems in section 2.

\section{Proof of the theorems}

We first prove theorem 2, assuming theorem 1 which we shall prove next.

Proof of theorem 2. Let $\left\{f_{n}(z)\right\}$ be the natural iterates of $f(z)$ where

$$
f(z)=z^{k}+a_{1} z^{k-1}+\cdots+a_{k}, k \geq 2 .
$$

We use the construction employed by Bhattacharyya and Arumaraj [1] for $F(f)$. We choose $R>0$ such that

$$
|f(z)|>R \text { for }|z|>R \text {. }
$$

We set

$$
D_{-n}=\left\{z:\left|f_{n}(z)\right| \leq R\right\}
$$

and define

$$
D=\cap_{n=0}^{\infty} D_{-n} .
$$

Then $F(f)=\partial D$.

From theorem 1 we have

$$
d\left(D_{-n}\right) \geq 2 R^{1 / k^{n}} \geq 2 \text { for every } n .
$$

Thus $d(D) \geq 2$, i.e. $d(F(f)) \geq 2$.

This proves the theorem.

Proof of theorem 1 . Take any constant $\eta$ such that $|\eta|=1$. We shall find this $\eta$ later. The equation

$$
\eta f(z)=f(\omega)
$$

defines a member of the function elements of $z=\infty$ which have the form

$$
\omega=\eta^{1 / k} z+b_{0}+\frac{b_{1}}{z}+\frac{b_{2}}{z^{2}}+\cdots
$$

one for each $k$-th root of $\eta$. Note that $\omega$ is not linear. Each such function element is a branch of an algebraic function $\omega$ of $z$ which satisfies (2.1). All possible branches which arise from analytic continuation are analytic for all finite $z$, except for a finite number of algebraic singularities and at these singularities and at these singularities the value of $\omega$ is finite since $\omega=\infty$ implies $z=\infty$. 
Denote by $g(z)$ any value of one of the algebraic branches obtained as above from (2.2). Then

$$
\eta f(z)=f(g(z))
$$

so that if $z$ is in $E$ i.e. $|f(z)| \leq R$ then

$$
|f(g(z))|=|\eta f(z)| \leq R
$$

i.e. $g(E) \subset E$ and $g(\partial E) \subset E$ as $\partial E$ is $\{z:|f(z)|=R\}$. Thus

$$
\{d(E)\}^{k} \geq \max _{z \in \partial E}|z-g(z)|^{k}
$$

i.e.

$$
\{d(E)\}^{k} \geq \max _{|f(z)|=R}\left\{R|z-g(z)|^{k} /|f(z)|\right\}
$$

Now

$$
\phi(z)=(z-g(z))^{k} / f(z)
$$

is analytic at $\infty$ with $\phi(\infty)=(1-\mu)^{k}$ where $\mu$ is the determination of $\eta^{1 / k}$ taken for $g(z)$ in (2.2). We choose $\eta=+1$ or -1 according as $k$ is even or odd, so that $\eta=(-1)^{k}$. Taking the determination $\mu=\eta^{1 / k}=-1$, gives $\phi(\infty)=2^{k}$ for the corresponding branch $g(z)$.

We now continue the branch of $g$ just defined to obtain all possible analytic continuations, keeping $z$ restricted to the domain $D=(C \cup\{\infty\}) \backslash E$. This gives rise to a possibly many valued (algebraic) function which has atmost finitely many algebraic singularities at certain finite points and a number of branches, each with a simple pole at $\infty$, where $g$ is as in (2.2).

The corresponding continuation of $\phi$ in (2.4) remains finite at finite points of $D$, since $f(z) \neq 0$ in $D$, while the values at $\infty$ are of the form $\left(1-\eta^{1 / k}\right)^{k}$, of which the greatest is $2^{k}$. Let the set of these continuations be denoted by $\phi^{*}$. If $\phi^{*}$ is not a constant, the values $\phi^{*}(D)$ form an open connected set by the open mapping theorem. The branches $\phi^{*}$ are continuous in $\bar{D}$, bounded at $z=\infty$, so in fact $\phi^{*}(D)$ is bounded, since $\phi^{*}(D)$ contains the value $2^{k}$.

Set

$$
\beta=\sup \left\{x \mid x>0, x \text { in } \phi^{*}(D)\right\}
$$

Then $\beta$ is $\phi(\gamma)$ for some $\gamma$ in $\partial D=\partial E$.

[For there exist $z_{n}$ in $D$, branches $\phi_{n}, \phi_{n}\left(z_{n}\right) \rightarrow \beta$. We can assume $z_{n}$ converges in $\bar{D}$ and $z_{n} \nrightarrow \infty$ since $\beta>2^{k}$.

Thus $z_{n} \rightarrow \infty$ in $\bar{D}$ and we can assume all $\phi_{n}$ are in fact the same 'branch' of $\phi$ since they are but finitely many. Thus $\phi(\gamma)=\beta$ is in the boundary of $\phi^{*}(D)$. By the open mapping theorem $\beta \in D$, so $\beta \in \partial D=\partial E=z:|f(z)|=R]$. 
Taking $z=\beta$ and fixing $g$ so that

$$
\left|\frac{\gamma-g(\gamma)}{f(\gamma)}\right|^{k}=\phi(\gamma)=\beta .
$$

We have in (2.3)

$$
d(E)^{k} \geq R \cdot \beta>2^{k} R .
$$

Thus

$$
d(E)>2 R^{1 / k} .
$$

The proof of the theorem is now complete.

Proof of theorem 3. Take $\phi=$ constant $2^{k}$ where $\phi$ is as in the proof of theorem 1. Consider the expansions near a zero of $f(z)$. Suppose that

$$
f(z)=a(z-\alpha)^{r}+\cdots,
$$

so $r \leq k$.

Then $z=g(z)$ at $z=\alpha$, so $g(\alpha)=\alpha$. By $(2.1)$

$$
\eta(z-\alpha)^{r}+\cdots=(g-\alpha)^{2}+\cdots
$$

so that

$$
g-\alpha=\eta^{1 / r}(z-\alpha)+\text { higher terms. }
$$

Hence

This gives

$$
z-g(z)=(z-\alpha)\left(1-\eta^{1 / r}\right)+\text { higher terms of }(z-\alpha) \text {. }
$$

$$
\begin{aligned}
2^{k} & =\frac{(z-g(z))^{k}}{f(z)} \\
& =(z-\alpha)^{k-r}\left(1-\eta^{k / r}\right)+\cdots, k-r \geq 0 .
\end{aligned}
$$

But then $k-r \leq 0$. Hence $r$ must be equal to $k$. This means that $f$ has a single zero of order $k$ at $\alpha$. Hence $f$ must be $(z-\alpha)^{k}$.

From Theorem 2 it follows that

$d(F(f))=2$ implies $f(z)=(z-\alpha)^{k}$ and conversely.

\section{References}

[1] Bhattacharyya, P., and Y.E. Arumaraj, On the Fatou Set of Monic Polynomials. Ann. Acad. Sci. Fenn. Ser A I Math. 7, 1982, 157-163.

[2] Brolin, H., Invariant set under iteration of rational function. - Ark. Mat. 6, 1965 -67, 103-144.

[3] Fatou, P., Sur les equations fonctionnelies. - Bull. Soc. Math. France 47, 1919, 161-271, and 48, 1920, 33-94, 208-314.

[4] Fatou, P., Surl'iteration des fonctions transcendantes entieres. - Acta. Math. 47, 1926, 337-370.

[5] Julia, G., Memoire sur l'iteration des fonctions rationelles. J. Math. Pures Appl. (8) 1, 1918, 47-245.

Indian Institute of Technology, Department of Mathematics, Madras-600 036, India. 